\title{
Expression of Concern: Knockdown of elF3d inhibits cell proliferation through G2/M phase arrest in non-small cell lung cancer
}

\section{Zhifeng Lin ${ }^{1} \cdot$ Liwen Xiong ${ }^{2} \cdot$ Qiang Lin ${ }^{1}$}

Published online: 18 August 2018

○) Springer Science+Business Media, LLC, part of Springer Nature 2018

\section{Expression of Concern: Med Oncol (2015) 32:183 \\ https://doi.org/0.1007/s12032-015-0625-8}

Concerns have been raised about this article [1] relating to the appropriateness of the use of the shRNA (5'-GCGGAG GGTTTGAAAGAATATCTCGAGATATTCTTTCAAACC CTCCGCTTTTTT-3') as a non-targeting control and similarities in text and formatting with other published articles. This is currently under investigation and appropriate editorial action will be taken once the investigation is concluded.
The authors did not respond to our correspondence regarding this expression of concern.

\section{Reference}

1. Lin Z, Xiong L, Lin Q. Knockdown of eIF3d inhibits cell proliferation through G2/M phase arrest in non-small cell lung cancer. Med Oncol. 2015;32:183.
The online version of the original article can be found under https ://doi.org/10.1007/s12032-015-0625-8.

\section{Qiang Lin}

LinqiangPhD@163.com

1 Department of Thoracic Surgery, Shanghai First People's, Hospital, Shanghai Jiaotong University, No. 100 HaiNing, Road, Shanghai 200080, China

2 Department of Pulmonary, Shanghai Chest Hospital, Shanghai Jiaotong University, 241 West Huaihai Road, Shanghai 200030, China 